\title{
EM 算法在假设检验中的应用*
}

\author{
房祥忠 ${ }^{* *}$ 陈家鼎 \\ (北京大学概率统计系, 北京大学数学与应用数学国家重点实验室, 北京 100871)
}

\begin{abstract}
摘要给出了 $\mathrm{EM}$ 算法在统计中一个新的应用领域——假设检验. EM 算法通常是 用来求解模型参数极大似然估计的一种有效的迭代算法. 这种算法利用数据扩张, 将 比较复杂的似然函数的最优化问题化成一系列比较简单的函数的优化问题. 而对于一 个模型来说，假设检验往往比极大似然估计更为复杂. 所以有必要找到简单的方法解 决假设检验中的较为复杂的优化问题. 所给出的方法不是建立在大样本理论基础上, 因此特别适合于中小样本情形.
\end{abstract}

\section{关键词 $\quad \mathrm{EM}$ 算法 假设检验 最大概率 小样本}

在解决假设检验问题时, 通常要找到一个统计量, 使得它在零假设下的确切或近似分布 与未知参数无关. 但是在模型比较复杂的情形下找到一个统计量的确切分布并不容易. 同时对 于中小样本来说, 利用近似分布得到的检验方法表现往往不好. 这些问题推动我们发展新的方 法.

本文首先利用 $\mathrm{EM}$ 算法 ${ }^{[1]}$ 给出求解最大概率值的方法，然后利用它寻找假设检验的临 界值和两类错误的概率. 特别地, 当这样做的时候, 不需要推导出统计量的精确的或渐近的分 布，因此这个方法容易实行. 另外，这个方法也可以用来计算通过大样本理论建立起来的检验 方法在某个固定样本量时的真实检验水平.

众所周知, $\mathrm{EM}$ 算法 ${ }^{[1]}$ 是用来对比较复杂的模型或数据求解极大似然估计的一种迭代算 法, 它将一个比较复杂的似然函数的最优化问题化成一系列比较简单的函数的优化问题. 近年 来出现了许多的变形以适应不同的情形 ${ }^{[2 \sim 5]}$. 在对 EM 算法和假设检验的研究过程中, 我们发 现可以将假设检验问题化成一个求解似然函数最大值的问题，从而可以利用 $\mathrm{EM}$ 算法将一个 比较复杂的假设检验问题化成一系列简单优化问题.

\section{1 关于最大概率的 EM 算法}

假定随机变量 $Y$ 服从概率分布 $P_{\theta}$, 这里 $\theta$ 在一参数空间 $\Theta$ 中取值. 随机变量 $Y$ 也许没 有被观测到, 被观测到的是它的一个已知函数 $Z=\mathcal{M}(Y)$. 作为特殊情形, $Z=Y$ 表示观测到 的就是 $Y$ 本身.

2002-07-04 收稿, 2002-12-12 收修改稿

* 国家自然科学基金资助项目 (批准号: 10071004)

** E-mail: xzfang@math.pku.edu.cn 
假定 $A$ 表示与 $Z$ 有关的随机事件. 举例来说, $A=\{R(Z)>r\}$, 这里 $R(Z)$ 是一个统计量 (比如似然比统计量), 而 $r$ 是实数. 这里所关心的是最大概率 $\sup \left\{P_{\theta}(A): \theta \in \Theta_{0}\right\}$, 这里 $\Theta_{0}$ 是 $\Theta$ 的子集. 在假设检验中，最大概率可以是真实的检验水平，也可以是犯第 1 类或第 2 类错误 的概率.

$\mathrm{EM}$ 算法是用来求解似然函数最大值点的工具，所以，如果能够将概率 $P_{\theta}(A)$ 看成似然函 数的值, 则可以利用 $\mathrm{EM}$ 算法得到最大概率 $\sup \left\{P_{\theta}(A): \theta \in \Theta_{0}\right\}$.

这个想法是可以实现的. 因为 $P_{\theta}(A)=P_{\theta}\{I(A)=1\}$, 所以概率 $P_{\theta}(A)$ 就是随机变量 $I(A)$ 的似然函数在 $I(A)=1$ 时的值, 这里 $I(A)$ 是随机事件 $A$ 的示性函数. 另外, $I(A)$ 是 $Z$ 的函 数从而也是 $Y$ 的函数. 即 $I(A)$ 可以看成 $Y$ 的删失变量, 故可以通过求取似然函数最大值的 EM 算法来求取最大概率的值，而且根据模型的特点可以选用任何一种合适类型的 EM 算法.

下面给出标准的 EM 算法, 并将其表述成关于求取最大概率的算法.

设 $f(y \mid \theta)$ 是 $Y$ 的密度函数. 从一个初始点 $\theta^{(0)} \in \Theta$ 开始, 则寻找 $\sup \left\{P_{\theta}(A): \theta \in \Theta_{0}\right\}$ 的 算法由下面的两步迭代而成 $(t=0,1, \cdots)$ :

$\mathrm{E}$ 步: 给定现在的值 $\theta^{(t)}$ 后, 对未知的对数似然函数 $l(\theta \mid Y)=\log f(Y \mid \theta)$ 求条件期望

$$
Q\left(\theta \mid \theta^{(t)}\right)=E_{\theta^{(t)}}\{l(\theta \mid Y) \mid A\} .
$$

$\mathrm{M}$ 步: 最大化函数 $Q\left(\theta \mid \theta^{(t)}\right)$, 求取最大值点 $\theta^{(t+1)}$ 作为下一步迭代的值, 即使得

$$
Q\left(\theta^{(t+1)} \mid \theta^{(t)}\right) \geqslant Q\left(\theta \mid \theta^{(t)}\right), \quad \theta \in \Theta_{0} .
$$

由 $\mathrm{EM}$ 算法的一般理论可以知道, $P_{\theta^{(t)}}(A)$ 关于 $t$ 是递增的. 如果选取适当的初值，则概 率 $P_{\theta^{(t)}}(A)$ 将收玫到最大概率 $\sup \left\{P_{\theta}(A): \theta \in \Theta_{0}\right\}$. 对于大的 $t$, 当序列 $P_{\theta^{(t)}}(A)$ 或 $\theta^{(t)}$ 变得稳 定以后, 则取 $P_{\theta^{(t)}}(A)$ 为最大概率 $\sup \left\{P_{\theta}(A): \theta \in \Theta_{0}\right\}$ 的近似值.

\section{2 假设检验}

这一节给出求取最大概率的方法在假设检验中的应用.

假设检验的拒绝域可以表示为 $A=(R(Z)>r)$ 的形式, 这里 $r$ 是实数, $R(Z)$ 可以是似然 比，也可以是其他统计量. 下面给出几个例子.

首先考虑最为一般的假设检验问题

$$
H_{0}: \theta \in \Theta_{0}, \quad H_{1}: \theta \in \Theta_{1},
$$

这里 $\Theta_{0}$ 和 $\Theta_{1}$ 都非空并且 $\Theta_{1}=\Theta-\Theta_{0}$. 令 $L(\theta, Z)$ 是似然函数, 似然比为

$$
R(Z)=\frac{\sup _{\theta \in \Theta} L(\theta, Z)}{\sup _{\theta \in \Theta_{0}} L(\theta, Z)}
$$

则这个检验的拒绝域应该具有形式 $A=(R(Z)>r)$.

再令 $g(\theta)$ 是在 $\Theta$ 的实值函数, $c$ 为一常数. 如果 $\Theta_{0}=(\theta: g(\theta) \leqslant c)$ 并且 $\Theta_{1}=(\theta: g(\theta)>$ $c)$, 则拒绝域应该具有形式 $A=(R(Z)>r)$, 这里 $R(Z)=g(\hat{\theta}), \hat{\theta}$ 是 $\theta$ 的极大似然估计或合适 的其他点估计.

最后, 统计量 $R(Z)$ 也是可以通过某些直观方法得到的统计量, 例如在列联表检验中所用 到的检验统计量.

好的检验方法应该选取 $r$ 尽可能的小. 对给定的水平 $\alpha$, 临界值是

$$
r_{\alpha}=\inf \left(r: \sup \left[P_{\theta}\{R(Z)>r\}: \theta \in \Theta_{0}\right] \leqslant \alpha\right),
$$


则拒绝域为 $A=\left\{R(Z)>r_{\alpha}\right\}$. 犯第 1 类错误的概率是 $\sup \left\{P_{\theta}(A): \theta \in \Theta_{0}\right\}$. 犯第 2 类错误的 概率是 $\sup \left\{P_{\theta}(\bar{A}): \theta \in \Theta_{1}\right\}$.

由于最大概率值 $\sup \left[P_{\theta}\{R(Z)>r\}: \theta \in \Theta_{0}\right]$ 是关于 $r$ 单调下降的, 既然概率 $P_{\theta}\{R(Z)>$ $r\}$ 对任何 $r$ 都可以求出, 所以临界值 $r_{\alpha}$ 可以通过二分法求得. 同时, 犯两类错误的概率也可 以通过求最大概率的方法得到.

\section{3 例子}

本节给出两个例子. 一个是检验列联表中因素是否独立, 另外的例子是利用组件级试验数 据检验成败型串联系统可靠度是否达到要求.

\section{1 列联表中的检验问题}

对于 $2 \times 2$ 列联表表 1 , 我们要检验两个变量的独立性.

表 1 入学成绩

\begin{tabular}{ccc}
\hline & 因素 $\mathrm{b}$ 水平 1 & 因素 $\mathrm{b}$ 水平 2 \\
\hline 因素 $\mathrm{a}$ 水平 1 & $n_{11}$ & $n_{12}$ \\
因素 $\mathrm{a}$ 水平 2 & $n_{21}$ & $n_{22}$ \\
\hline
\end{tabular}

表 1 中 $n_{i j}(\geqslant 0)(i, j=1,2)$ 是观测到 的个体数. 个体的总数目 $N=\sum_{i, j=1}^{2} n_{i j}$ 是一个固定的数.

在这个例子中, 没有删失数据, 这 时 $Y=Z=\left(n_{11}, n_{12}, n_{21}, n_{22}\right)$. 但是 却难于发现确切分布不依赖于未知参数的量. 人们通常应用近似方法——卡方检验法 ${ }^{[6,7]}$ : 设

$$
R=\sum_{i, i=1}^{m} \frac{\left(n_{i j}-n_{i} \cdot n_{\cdot j} / N\right)^{2}}{n_{i} \cdot n_{\cdot j} / N},
$$

这里 $n_{i}=\sum_{j=1}^{2} n_{i j}(i=1,2)$ 是按行的个体数, 而 $n_{\cdot j}=\sum_{i=1}^{2} n_{i j}(j=1,2)$ 是按列的个体 数, 则当 $N$ 比较大时, $R$ 具有自由度为 1 的卡方分布. 对给定的检验水平 $\alpha$, 临界值 $\chi_{1, \alpha}^{2}$ 可以 取为使卡方统计量大于 $\chi_{1, \alpha}^{2}$ 的概率恰好为 $\alpha$. 如果 $R$ 大于 $\chi_{1, \alpha}^{2}$, 则应该拒绝独立性的假设.

但是如果 $N$ 不足够大，则对于临界值 $\chi_{1, \alpha}^{2}$ ，检验的真实水平可能不是 $\alpha$. 我们可以利用 $\mathrm{EM}$ 算法来计算它的真实水平. 即计算概率最大值

$$
\sup \left[P_{p_{a} p_{b}}\left(R>\chi_{1, \alpha}^{2}\right): 0<p_{a}, p_{b}<1\right],
$$

这里关于概率 $P_{p_{a} p_{b}}(\cdot),\left(n_{11}, n_{12}, n_{21}, n_{22}\right)$ 具有多项分布, 总频数为 $N$, 单元概率为

$$
\left[p_{a} p_{b}, p_{a}\left(1-p_{b}\right),\left(1-p_{a}\right) p_{b},\left(1-p_{a}\right)\left(1-p_{b}\right)\right] .
$$

对数似然函数为

$$
l=n_{1} \cdot \log \left(p_{a}\right)+n_{2} \cdot \log \left(1-p_{a}\right)+n_{\cdot 1} \log \left(p_{b}\right)+n_{\cdot 2} \log \left(1-p_{b}\right)+c,
$$

这里 $c$ 是不依赖于参数 $p_{a}$ 和 $p_{b}$ 的常数.

从这里出发, 对于给定的初值 $\left(p_{a}^{(0)}, p_{b}^{(0)}\right)$, 可以知道 EM 算法的两步迭代在这里变成简单 的形式:

$$
\begin{gathered}
p_{a}^{(k+1)}=E_{p_{a}^{(k)} p_{b}^{(k)}}\left(n_{1 \cdot} \mid R>\chi_{1, \alpha}^{2}\right) / N, \\
p_{b}^{(k+1)}=E_{p_{a}^{(k)} p_{b}^{(k)}}\left(n \cdot 1 \mid R>\chi_{1, \alpha}^{2}\right) / N, \quad k=0,1, \cdots .
\end{gathered}
$$

在序列 $\left(p_{a}^{(k)}, p_{b}^{(k)}\right)$ 变得平稳之后, 取概率 $P_{p_{a}^{(k)}} p_{b}^{(k)}\left(R>\chi_{1, \alpha}^{2}\right)$ 为最大概率值的近似值. 这 个过程应该选取不同的初值多次重复. 最后的概率最大值就作为检验的真实水平.

对于给定的 $\alpha$, 可以利用公式 (2.2) 找到临界值 $r_{\alpha}$. 
下面给出一个计算实例. 通过这个简单的例子说明我们的方法.

某校数学系 2002 年研究生入学考试共有 47 名考生参加. 表 2 给出各种情形下的人数. 数学是指 3 门数学课的分数之和, 政英表示政治和英语的分数之和. 高分表示成绩在平均值以 上，低分表示成绩不超过平均值. 我们试图检验政英成绩和数学成绩是否具有独立性.

对于表 2 的数据, $R$ 的值为 10.7288. 对应 于检验水平 0.05 和 0.01 卡方检验的临界值 分别为 3.84146 和 6.63490. 根据卡方检验, 无论是按照水平 0.05 还是 0.01 , 均应当拒绝

\begin{tabular}{|c|c|c|}
\hline & 数学高分 & 数学低分 \\
\hline 政英高分 & 21 & 6 \\
\hline 政英低分 & 6 & 14 \\
\hline
\end{tabular}
政英和数学的独立性.

但是由 $\mathrm{EM}$ 算法, 我们可以算出对于临界值 3.84146 和 6.63490 的真实的检验水平分别为 0.069456 和 0.045556 , 所以名义水平与真实水平之间有比较大的差别.

对应于检验水平 0.05 和 0.01 , 我们利用 $\mathrm{EM}$ 方法求临界值. 但是由于 $R$ 是离散变量, 找 不到临界值使得它的真实水平恰好就是 0.05 或 0.01 . 对于水平 0.01 最为接近的是 0.010091 , 对 应的临界值是 22.98913 . 对于水平 0.05 最为接近的是 0.047322 , 对应的临界值是 4.61802 . 因为 $R=10.7288<22.98913$, 所以在 0.01 的检验水平下，不能够否定政英成绩与数学成绩的独立 性.

\section{2 利用组件级数据对串联系统可靠度的检验}

在科研生产中, 经常要研制比较复杂的系统. 由于整个系统的造价昂贵, 经常要根据系统 的组件试验数据对整个系统的可靠度进行评估.

设一系统由 $m$ 个成败型单元串联组成, 第 $i$ 个单元的可靠度为 $p_{i}$, 则系统的可靠度为

$$
p=p_{1} p_{2} \cdots p_{m} .
$$

对第 $i$ 个单元进行了 $n_{i}$ 次试验, 成功次数为 $s_{i}$. 下面利用 $\left(n_{i}, s_{i}\right)(i=1,2, \cdots, m)$ 对系统可靠 度 $p$ 进行检验. 检验的问题是

$$
H_{0}: p<p^{*}, \quad H_{1}: p \geqslant p^{*},
$$

这里 $p^{*}$ 是对系统的可靠度的最低要求, 是事先给定的值.

可以知道 $p$ 的极大似然估计为

$$
\hat{p}=\frac{s_{1}}{n_{1}} \frac{s_{2}}{n_{2}} \cdots \frac{s_{m}}{n_{m}} .
$$

对给定的检验水平 $\alpha$, 可以利用第 2 节的方法找到形式为 $A=\left(\hat{p}>r_{\alpha}\right)$ 的否定域. 对于具 体的 $r$, 利用 $\mathrm{EM}$ 算法给出求解 $\sup \left[P_{p_{1}, \cdots, p_{m}}\{\hat{p}>r\}: 0 \leqslant p_{i} \leqslant 1, i=1, \cdots, m\right]$ 的方法, 然后采 用二分法求出 $(2.2)$ 式中定义的临界值 $r_{\alpha}$.

在数据 $\left(n_{i}, s_{i}\right)(i=1,2, \cdots, m)$ 下的对数似然函数为

$$
\log L=\sum_{i=1}^{m} s_{i} \log p_{i}+\left(n-s_{i}\right) \log \left(1-p_{i}\right)+c,
$$

这里 $c$ 是与 $p_{1}, \cdots, p_{m}$ 无关的常数.

给定 $\left(p_{1}, \cdots, p_{m}\right)$ 的一个初值 $\left(p_{1}^{(0)}, \cdots, p_{m}^{(0)}\right)$, 则在已知 $l$ 步迭代后, $\mathrm{EM}$ 算法的 $\mathrm{E}$ 步是

$$
Q\left(p_{1}, \cdots, p_{m}, p_{1}^{(l)}, \cdots, p_{m}^{(l)}\right)=\sum_{i=1}^{m} s_{i}^{(l)} \log p_{i}+\left(n-s_{i}^{(l)}\right) \log \left(1-p_{i}\right)+c,
$$

这里 $s_{i}^{(l)}=E_{p_{1}^{(l)} \ldots p_{m}^{(l)}}\left(s_{i} \mid \hat{p}>r\right)$. 
$\mathrm{EM}$ 算法的 $M$ 步是在 $p=p_{1} \cdots p_{m}=p^{*}$ 下求出 $Q\left(p_{1}, \cdots, p_{m}, p_{1}^{(l)}, \cdots, p_{m}^{(l)}\right)$ 关于 $\left(p_{1}, \cdots, p_{m}\right)$ 的最大值点.

利用 Lagrange 乘子法得到最大值点为

$$
p_{i}^{(l+1)}=\frac{s_{i}^{(l)}+\lambda}{n_{i}+\lambda}, \quad i=1,2, \cdots, m,
$$

其中 $\lambda$ 是方程 (方程的左端是关于 $\lambda$ 严格单调增函数)

$$
\prod_{i=1}^{m} \frac{s_{i}^{(l)}+\lambda}{n_{i}+\lambda}=p^{*}, \quad \lambda>-\min \left\{\widetilde{s}_{i}, i=1,2, \cdots, m\right\}
$$

的解.

这样得到一个序列 $\left\{\left(p_{1}^{(l)}, \cdots, p_{m}^{(l)}\right), l=1,2, \cdots\right\}$. 根据 $\mathrm{EM}$ 算法的一般原则, 这个序列使得 $P_{p_{1}^{(l)}, \cdots, p_{m}^{(l)}}\{\hat{p}>r\}$ 是单调不减的. 如果初值选取恰当, 则 $P_{p_{1}^{(l)}, \cdots, p_{m}^{(l)}}\{\hat{p}>r\}$ 收玫到 $P_{p_{1}, \cdots, p_{m}}\{\hat{p}>$ $r\}$ 的最大值. 我们的计算例子表明，对于这个模型，收玫值不受初值的影响，但选取得好，则 收玫得较快.

设一串联系统由 4 个单元串联而成. 4 个单元的试验数和成功数分别为试验数据 $(35,34)$, $(40,39),(55,54),(60,60)$. 检验

$$
H_{0}: p<0.80, \quad H_{1}: p \geqslant 0.80,
$$

可以求出系统可靠度的极大似然估计为

$$
\hat{p}=\frac{34}{35} \frac{39}{40} \frac{54}{55} \frac{60}{60}=0.929922,
$$

对应于检验水平 0.05 和 0.01 的临界值分别为 0.898572 和 0.932728 . 由于 $0.898572<0.929922<$ 0.932728 , 所以在 0.05 的水平下应该否定原假设，但是在 0.01 的水平下应该不能否定原假设.

\section{4 讨论}

本文方法在中小样本时是有用的，有时同求极大似然估计的 EM 算法，Monte-Carlo 模拟 可以应用到 $\mathrm{E}$ 步的条件期望中，因为这里的条件事件 $A$ 的概率总是正的，故不用求出条件分 布的解析式. 条件期望也可以通过模拟直接得出来，在这种观点下，求解最大概率时的 EM 算 法比求解极大似然估计的 EM 算法更容易操作.如果所求概率值需要模拟，则对样本量比较大 的情形会需要比较多的运行时间.

\section{参考文献}

1 Dempster A P, Laird N M, Rubin D B. Maximum likelihood from incomplete data via EM algorithm (with discussion). J R Statist Soc B, 1977, 39(1): 1 38

2 Fessler J A, Hero III A O. Space alternating generalized expection maximization algorithm. IEEE Trans Siginal Process, 1994, 42(10): 2664 2677

3 Liu C, Rubin D B. The ECME algorithm: a simple extension of EM and ECM with fast monotone convergence. Biometrika, 1994, 81(4): 633 648

4 Meng X L, Dyk V D. The EM algorithm_—an old folk-song sung to a fast new tune. J R Statist Soc B, 1997, 59(3): 511 567

5 Meng X L. Fast EM-type implementations for mixed effects models. J R Statist Soc B, 1998, 60(3): 559 578

6 Lioyd C J. Statistical Analysis of Categorical Data. New York: John Wiley and Sons, 1999. 125 127

7 Spiegel M R. Schaum's Outline of Theory and Problem of Statistics. New York: McGrew-Hill Companies, 1988. $245 \sim 249$ 